\title{
Design Research of Machine Frame on Three Dimensional Models
}

\author{
Zhanjun Liu \\ Faculty of Aerospace Engineering \\ Shenyang Aerospace University \\ Shenyang, China \\ Liuzhanjundef1@163.com
}

\begin{abstract}
The present paper takes some machine frames as the object of study, three dimensional model is based on the CATIA software, the model and the hypothesized assembly are carried by the CATIA software. It is firstly introduced the aviation manufacture project development by proposed new request, the CATIA software formidable function has provided the solution to this kind of demand. Meets down is carried on the structure technology capability analysis to some machine frame, it is given by several kind of jig design proposal, and through the efficient feasibility analysis, it determines the design of assembly jig. Finally, it has finished the assembly process dynamic simulation and the efficient analysis, which has realized the goal of the assembly jig rated capacity by using the CATIA software.
\end{abstract}

Keywords-assembly type; CATIA; three dimensional modeling; feasibility analysis; dynamic simulation

\section{INTRODUCTION}

As a kind of their own power to create lift to support its own special machine in the air, the product is not only appearance strict pneumatic requirements, but internal mechanism is complex, with a huge number, system and complex parts tightly, technical indicators and require higher characteristic, and the mechanization and automation degree of assembly installation work are lower, manual labor accounts for a large proportion, lowly labor productivity. The aircraft assembly and installation are of high quality requirements, technical difficulty is big, to improve the level of aircraft assembly and installation technology [1], it is of great significance in aircraft manufacturing. So the aircraft assembly quality becomes the bottleneck of improving product accuracy. Aircraft manufacturing accuracy depends largely on the accuracy of the assembly, and it needs to use many of the standard technology and equipment, to ensure the assembling between the frame and the coordination. As a result, the design guarantees the coordination between the assembly jig method, which is peculiar to the aircraft manufacturing technology. Machine frame is horizontal stress component on the fuselage, as the ring is stiffener, besides keeping body shape and aerodynamic shape, it is more important to the function of the plane under concentrated load passed to the fuselage skin [2].

\section{The MACHINE Frame StRUCtURE AND MANUFACTURABILITY ANALYSIS}

Fuselage frame and the wing ribs are similar, it is a lateral force component on the fuselage, which has to keep the fuselage appearance, support the skin, improve the stability of the skin, to facilitate the role of withstanding local aerodynamic load. Therefore, its main role is under concentrated load and passed on to the fuselage skin. So it is a balance of plane structure under the effect of concentration and distribution of shear flow.

Machine frame is in the body section, for the riveting combo box to made up of top, bottom and side frame edge. Panels are made of the thick plate, strengthening material is on the web of box used to strengthen the stiffness of the frame [3]. Due to the continuous development of aircraft design and manufacturing technology, the structure of the increasing load per unit area is increasing, in order to reduce the structure weight, improving the integrity of aircraft structure is the inevitable development trend, by given the size and cost of many factors, frame is still common ring frame structure at present. Its structure is shown in Fig. 1.
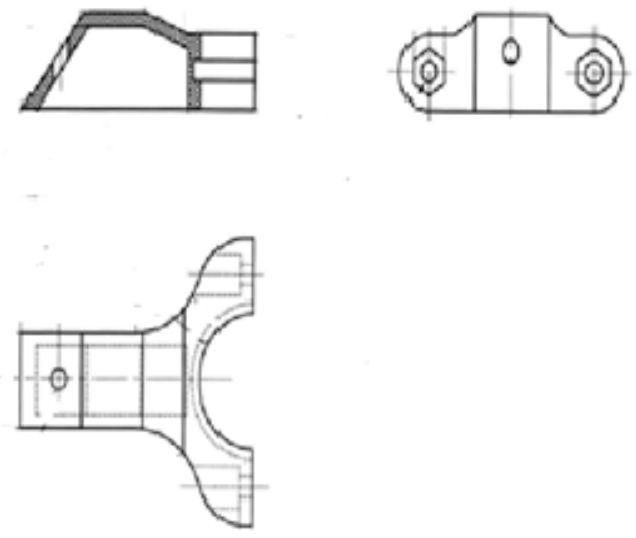

Figure 1. The mold style for frame structure

Machine frame can be divided into frame edge of four parts on the process. After divided, raw materials and the existing technology level reduce the production conditions of the requirements, which has an important effect of reducing the manufacturing cost [4]. Assembly is assembled according to each part in turn, which can make the assembly work orderly, but also make the whole box type in shelf distribution balance, and do not make large frame plate and other material deformation, to impact the whole assembly accuracy and 
coordination, at the same time, to facilitate periodic inspection after assembly. Before submitting inspection, assembly should be good on the machine frame all debris removal. Every batch is weighing out a box. The acceptance of box should be labelled and certificate.

Machine frame is the important parts of the aircraft, it is in the fuselage section, since it has to keep the fuselage shape, maintaining the stability of the skin effect, therefore, it is of high shape accuracy requirements, relationship to the aerodynamic shape of the aircraft, which is related to the aircraft's flight performance [5]. The particularity of machine frame structure, the location of the high degree requirements and the surface of the high accuracy requirement meet the requirements of coordination, so in the design of machine box, it is given by assembly jig design solutions, and through the analysis on the feasibility and economy of several alternatives, eventually it determines a solution as the final result. Assembly jig design principles are usability. Positioning is reasonable, compacted and reliable, workers are conveniently in operation. Positioning and clamping parts are of simple operation. The design of the positioning system should guarantee the coordination technology, equipment and reasonable manufacturing coordination method. Stiffness is reasonable and important components should eliminate stress. The use of activity is to locate a position relatively stable, the tooling structure design should eliminate or reduce the temperature on the impact of factors of coordinating by the corresponding measures. On the premise of meeting the requirement, the structure of the process equipment should be low cost. Process equipment structure of similar products is unification, as far as possible to facilitate the manufacture of process equipment. In the process of product positioning and compaction, it should has the necessary protection measures, to prevent scratch products [6]. In active area, operators must not have sharp corners or sharp edges, it is paied attention to the use of advanced structure and advanced technology method, in order to improve the usability of equipment, process equipment and reduce manufacturing costs. For the assembly of the machine frame type choice, it is conceived of the following two schemes in the design, the skeleton of the frame is the focus of the design. Skeleton includes frame type, combined type, distributed and overall base type. Skeleton frame type is used for the frame, ribs, girders, such as the plane shape of assembly, plate, and small three-dimensional assembly, the modular framework includes general by base, pillar, arms beam, and standardized component. The characteristics of distributed skeleton type are no overall skeleton, various positioning clamping piece is in fixed workshop foundation based metal skeleton. The overall base frame has an integral part of the base, more adjustable bearing support is on the shop floor. According to the structure characteristics of the machine frame, the choice first is determined as a skeleton frame type, but the frame type is divided into vertical feed type frame and the rotation type frame peaceful type framework. This framework can be bolted on the basis of the special, which also can be directly placed on the floor [7]. This framework is composed of channel steel welded frame, the frame is with rotating connection on the base of the bearing. Advantages are firstly to keep upright type frame cover in area, which can be carried out on the size of the component assembly, secondly, it can be used at the bottom of the adjustable bearing and not fixed with the floor, when foundation is deformation, it can be adjusted at any time. Disadvantages are the combination of machine frame for planar shape, and belongs to the class, the frame requires a higher surface aerodynamic shape, and this solution is for parts only used when assembling a positioning and clamping parts, which cannot achieve the requirements of the surface accuracy. When it is assembled artifacts in a vertical position, it must firstly be fixed workpiece, which can't place on the skeleton, workers can't guarantee most of the work in the most advantageous position. The rotation type framework covers smaller area, it can be firstly placed the frame level, and then fixing the workpiece clamping, to rotate the framework for the vertical state, workers can be adjusted according to oneself circumstance [8]. In the end, the rotating type framework is more commonly used card board assembly, flexible bar card board can also be relatively free to move on the skeleton, and it easy to leave work to take beneficial. Faults framework itself is required channel steel, increasing the quality of the frame.

\section{The CoORdinAtion Scheme Design}

Aircraft contains parts processing to parts assembly after many production processes, each link will produce manufacturing error. In order to guarantee the manufacturing and coordination accuracy, it must ensure that the large number of manufacturing accuracy of the process equipment itself and related technology and equipment coordination between the accuracy basic requirements, for design coordination route is that guarantee aircraft parts, assembly, interchangeability of parts and components, and that ensure their geometric parameters, appearance, joint and separated the interchangeability. At the same time, the coordination route design should be met, they are minimum that process equipment, parts of the shape, size of the transmission link data, the amount of error and the link. The Coordinate system basically is that line template, standard sample model, the local coordinate system, the line standard sample coordinate system and the application of computer aided design and manufacturing technology system. Firstly, model standard samples for the line coordinate system can be made on the surface of the modalities parts with the higher coordination, and which is easy to check. But manufacturing standard sample cycle is long, high technical requirements [9]. Secondly, die line template of local standard samples are on the surface of the coordinate system to ensure the modalities coordination, which can only make local standard samples, save the cost of most manufacturing standard sample, which improve the productivity. Finally, the computer aided design and manufacturing technology of coordinate system, and in the former there is big difference between two coordinate systems, it can be made of independent method, through the establishment of a unified precision plane geometry database, shape and internal structure of the plane geometry information, it passed directly to the data equipment, aircraft parts, and machining process equipment. The assembly of the machine frame is using computer aided design and manufacturing technology of coordinate system, the digital quantity coordination method. Firstly it is to establish a digital frame according to the theory of graph model, and with interference analysis, there is no interference of components imported CNC 
machine tools. For joint angle box, it can be directly used to machining processing. For sheet metal parts of the assembly of standard samples, the need is to make a lot of molding, assembly type frame board and card board.

\section{The Machine Frame Assembly of Process PLANNING}

First of all, with forming a complete set of spare parts, the typical procedure check the surface quality, whether there are a scratch and burr etc, checking parts size tolerance is in line with the requirements, examining the corresponding assembly fixture ensure its accuracy. Secondly, according to the plane drawings to determine the assembly parts list, it is including the name of the parts, parts size and quantity, it is fixing the aircraft parts and assembly, assembly should be according to the district assembly, this is the assembly work orderly. Finally, checking assembly parts is including the riveting quality inspection, drilling row nest quality inspection accuracy checking and the position of the parts.

\section{The Jig Design of Machine Frame Assembly}

According to the plane drawings, the design basis is for the horizontal axis of the plane symmetric axis, so the horizontal axis of symmetry axis is as the aircraft type and aircraft design reference, so that it can avoid datum transformation with multifarious calculation, also eliminating was caused by the error accumulation of datum transformation. The Box is on the location of the various parts of the reference line and plane level symmetry axis is as the benchmark, through the comprehensive research of drawings and technical process, it is selected the horizontal axis and axis symmetry aircraft as box type rack design benchmark, which is accurate, simple and reasonable.

Assembly type frame is composed of the following sections that frame and location, the type of substrate is used for fixed and support of the clamping and positioning bear weight of parts in the assembly process, and it can ensure the accuracy and stability of the spatial location of components, type rack main working components is used to ensure the workpiece with the accurate position in the assembly process, it is to make the workpiece firmly down on the locations of strength element. Auxiliary equipment is including job pedal, ladder, bracket, workbench, hoisting hanging, trucks and lighting equipment. The assembling rack must have a higher position accuracy, if the location of the frame is errors, then the assembly good product must also have the error, the accuracy of location has ground level degree, skeleton frame of manufacturing accuracy and so on. The assembly jig design can improve the work efficiency. The frame structure simplify, components should be standardized. Place state machine is in the frame of the box. Artifacts should make workers under the most favorable work attitude, it should make the most of the operation under the standing posture, within the scope of work, in addition, it also should consider to saving workshop area, after comprehensive consideration for box in the placement of assembly, type rack status should be used positioning clamping in horizontal, when assembling, it can rotate according to specific circumstances, for the workers is in the best working state.
Machine frame selection is positioning clamping piece and layout. Positioning is the main work frame element, which is used to ensure the workpiece with the accurate position in the assembly process. Clamping piece is to make the workpiece firmly down on the locations of strength components. Since aircraft airframe, thin walled structure is generally rigid, insufficient rigidity. Locating a select box assembly jig is that the butterfly positioning, positioning support as well as internal and external card board. Clamping piece requirement is clamping reliable, which is easy to operate, it don't damage the workpiece. The frame with flanging on edge article adopts dynamic spiral compression device, it will frame plate and flange, wild card board. With no edges of workpiece, it is using dynamic spiral compression device, which is combined with locations of frame plate fixation. Machine the box is out of the way. Artifacts within the frame assembly are one of the main problems in frame structure scheme, a greater influence is a good choice on the type of frame structure, which can simplify the structure of the frame, the safety does not damage the workpiece, which can also save the factory workshop area, to simplify handling equipment. For smaller artifacts, the frame is simple, as long as the positioning clamping parts can shrink enough size, which can remove the artifacts. For large size components, especially large, large aircraft parts should be seriously considered. Large size components generally there are three kinds of frame modes, from type rack above the frame, the longitudinal frame, side frame. For box, the type is in the complete assembly of the shelf after assembly work, every positioning clamping piece will open the bolt of card board inside and outside and is back away from the box, and then remove the box from the assembly jig to reverse course. The role of the wild card board is the guarantee of the outside of the box shape, in order to guarantee the assembly of the frame's appearance and shape the consistency of the theory, and the longer is the length of contact card board box, which is to guarantee the assembly frame's appearance the better, but considering the frame assembly must be from the assembly jig to smooth out problems, the design of card board can't be closed, and it should have certain mobile clearance. Card board, however, is too long, which can bring inconvenience to the use of the frame, and made of card board material, the manufacturing cost, increasing the assembly jig also increases the quality of the frame. Card board is very flexible in use, the production of card board material will be less. In consideration, opening a hole on wild card board is with bolt pressure pushing away from the structure, which is to make the card board move freely, convenient location or artifacts. With a block, the skeleton is linked together, which is easy to locate. The placement of card board is inside the form. Similarly, in the right box, the card board is still hold-down bolts activities away from institutions, therefore, under the precondition of dowel meet its positioning, bolts pressure should be fixed, obviously, it is very favorable to enhance the accuracy of the assembly.

The shape of the skeleton and placement form role is to support card board, positioning device, clamping fixture box and all the assembly of the parts. According to simple calculations, it choose the channel steel, for carrying the weight of the main frame, the two channel steel is butt welding, the rest of the auxiliary support are the channel steel and the main skeleton butt. The determination of bearing is the connection 
with the skeleton. According to the box parts drawings and assembly type of aircraft scheme analysis, the bearing assembly jig is selecting the type of medium-sized bearings. Choosing the cause of the pillar is fewer materials, manufacturing easy, which can fully support the whole frame, and guarantee the stability of assembly process. Pillar and the skeleton use standard parts with rotor rotating device to connect, which selects the index plate bearing connected to the skeleton, the fixed the rotor is by connecting welding, metal sheet and the skeleton rotational part connected with the bearing.

\section{Molding Technologies of Relative Position IS WITH FLARING RATIO}

The influence is pipe position when the end of the pipe is beyond $10 \mathrm{~mm}$, the magnetic pressure is almost zero. When the coil is no taper, it won't make the pipe deformation. When the Lc is negative, the tube is expanding into a cone. Pipe relations of relative position is with the flaring ratio. Regardless of the charging energy [10], when Lc/R is 0.5 , pipe belling is the most. When $\mathrm{Lc} / \mathrm{R}$ is less than 0.5 , the pipe belling ratio decreases with the decrease of the $L c / R$, when $L c / R$ is greater than 0.5 , and which decreases due to the distance between the tube and coil, the magnetic pressure is of acting on the pipe, thus it reducing the flaring. As a tapered coil is zero, due to the distance between the tube and coil, the basic constant is flaring.

The tapered pipe forming length is equal to zero by the coil in flaring, no matter how high, forming length is under the same discharge energy, tube and pipe near the clamping frame deformation is nearly the same, and the middle part is in the shape of straight uniform flaring, such as using coil with a taper shape, regardless of the length of the forming, the pipe is clamping frame, which is the same as in other parts of the deformation.

In the electromagnetic forming process, most of the deformation is in pulse pressure disappears. Under the effect of pulse pressure, the metal is obtained great acceleration and got a lot of kinetic energy, metal forming mainly depends on the kinetic energy to achieve. The electromagnetic forming is with pure electromagnetic properties, its forming process is not affected by the mechanical inertia of the moving parts, so the electromagnetic forming can achieve rapid processing, which can work hundreds of times per minute. And the intensity of magnetic pulse can be accurately controlled, which is easy to realize mechanization and automation [10].

\section{CONCLUSIONS}

The present paper takes some machine frames as the object of study, three dimensional model is based on the CATIA software, the model and the hypothesized assembly are carried by the CATIA software. It is firstly introduced the aviation manufacture project development by proposed new request, the CATIA software formidable function has provided the solution to this kind of demand. Meets down is carried on the structure technology capability analysis to some machine frame, it is given by several kind of jig design proposal, and through the efficient feasibility analysis, it determines the design of assembly jig. Finally, it has finished the assembly process dynamic simulation and the efficient analysis, which has realized the goal of the assembly jig rated capacity by using the CATIA software.

\section{REFERENCES}

[1] A. Barr, and E.A. Feigenbaum, "The Handbook of Artificial Intelligence”, California Vol. II Heurostech Press New York, pp. 17-21, 1982.

[2] P. H. Winstone and B. K. P. Horn, “LIST. U.A.” Addison-Wesley Publishing Company, London, pp. 23-25, 1984.

[3] M. White Frank, "Fluid mechanics (third edition)," McGraw-Hill Inc, NJ, Highstown, pp. 221 -222, 1994.

[4] S. Middleman, "An introduction to fluid dynamics, "John Wiley and Sons Inc, New York, pp. 16-17, 1998.

[5] Z. J. Liu, "Difficulty reliability prediction research of titanium plate stamping forming based on the fuzzy control,” Plast. Eng. J. Beijing, pp. 63-66, 2005.

[6] J. Feng, "The Mixture of The Multimode System Reliability Growth Model,” Comput. Appl. Res. Supplement, pp. 60-61, 2003.

[7] Y. X. Xu. "Complex Mechanical Fault Diagnosis Method of Fractal and Wavelet,” Mechanical Industry Publishing House, Beijing, pp. 24-25, 2005.

[8] C. X. Weng, “Reliability Growth,” Science Press, Beijing, pp. 130-131, 1993.

[9] F. Guo, "Reliability Data Collection And Analysis,” Nat. Defence Ind. Press, p. 22, 1995.

[10] W. D. Wen. "Prediction model based on the fuzzy reliability," Aircraft Engine, Beijing, pp. 322-323, 2003. 